\title{
Penetrating keratoplasty in Africa: graft survival and visual outcome
}

\author{
David Yorston, Mark Wood, Allen Foster
}

\begin{abstract}
Aim-To study the survival and visual outcome of penetrating keratoplasty in an African setting.
\end{abstract}

Methods-A retrospective analysis of 216 corneal grafts, performed on 203 eyes of 186 patients, at Kikuyu Hospital, Kenya over a 5 year period.

Results-Half of the transplants were carried out for keratoconus with only $5 \%$ of the grafts being undertaken for corneal scarring caused by trachoma or measles. The average follow up was $\mathbf{2 7 . 3}$ months. The probability of graft survival at 2 years was $87.4 \%$ (95\% CI 80.6\%-94.3\%) for keratoconus and $64.7 \%$ (95\% CI 54.8\%-74.6\%) for other corneal pathologies. Forty seven grafts $(21.8 \%)$ in 36 patients $(17.7 \%)$ are known to have become opaque. The commonest causes of graft opacification were bacterial keratitis $(6.0 \%)$, endothelial failure $(6.0 \%)$, and graft rejection (5.1\%). Preoperatively $55 \%$ of keratoconus eyes and $75.7 \%$ of non-keratoconus eyes were blind. Postoperatively, $5 \%$ of keratoconus eyes and $41.7 \%$ of the nonkeratoconus eyes were blind. Normal vision was achieved in $53.7 \%$ of operated eyes. Grafts carried out for keratoconus had a better visual outcome than grafts performed for other corneal pathologies. Preoperatively, $12.4 \%$ of keratoconus and 48.5\% of non-keratoconus patients were blind in their better eye. Postoperatively, $1.1 \%$ of keratoconus patients and $25.7 \%$ of non-keratoconus patients were blind. The number of patients with normal vision in the better eye increased from $32(17.2 \%)$ to $106(57.0 \%)$. Sight was restored to 34 blind patients, but two patients with severe visual impairment preoperatively were blind at their last follow up. There was therefore a net reduction of 32 in the number of blind patients after 216 keratoplasties.

Conclusions-Penetrating keratoplasty can be successful in Africa, particularly for keratoconus and other corneal dystrophies. However, penetrating keratoplasty has a limited role in the treatment of blindness from corneal scarring due to trachoma, measles, and vitamin $A$ deficiency for which community based preventive measures must remain the priority.

(Br F Ophthalmol 1996;80:890-894)

Correspondence to: Dr Allen Foster, Institute of Ophthalmology, 11, Bath Street, London ECIV 9EI

Accepted for publication 12 July 1996

The prognosis for penetrating keratoplasty in the industrialised world has improved greatly during the past 20 years. However, relatively little is known about the effectiveness and role of penetrating keratoplasty in countries of the developing world, particularly sub-Saharan Africa. ${ }^{1}$ In the UK, corneal blindness accounts for $2 \%$ of blind registrations, ${ }^{2}$ while in Africa corneal disease is responsible for at least $25 \%$ of all blindness. ${ }^{3}$ Studies have shown that the most important causes of corneal blindness in Africa are vitamin A deficiency associated with measles and trachoma, diseases which are amenable to preventive measures. ${ }^{3-6}$ Unfortunately many of the eyes blinded by these conditions are unsuitable for keratoplasty. This study investigates the indications and outcome of keratoplasty in Africa.

\section{Methods}

A retrospective review of the records of all patients who received a corneal graft at Kikuyu Hospital in Kenya before 1 January 1993 was undertaken in January 1994. All patients no longer attending the clinic were written to at their last known address and invited to attend for an eye examination.

The measurements of outcome were graft transparency, visual acuity in the operated eye, and visual acuity in the better eye of the patient. The method of Kaplan and Meier, for estimating survival from incomplete data, was used to analyse the probability of graft survival. Visual acuity was classified according to the World Health Organisation's recommended categories of visual loss. ${ }^{7}$ Blindness is defined as a vision of less than $3 / 60$ to NPL. The term visual acuity is used to refer to the vision in an eye. The term visual status is used to refer to the vision of the patient (that is, vision in the better eye).

\section{Results}

DEMOGRAPHICS

Altogether 216 grafts were carried out on 203 eyes of 186 patients during the period under review. Fifteen eyes had more than one graft. Of the 89 keratoconus patients, $61.8 \%$ were male, compared with $51.5 \%$ of 97 nonkeratoconus patients $(p>0.1)$. The mean age for the keratoconus grafts was 17.8 years $(95 \%$ confidence interval (CI) 15.8-19.8) compared with 43.3 years (95\% CI 38.9-47.7) for the non-keratoconus patients.

DIAGNOSES

The preoperative diagnoses for all 216 penetrating keratoplasties are shown in Table 1. 
and herpetic disease $8 \%$. Of 14 repeat grafts for graft failure nine were for keratoconus.

\section{SURGERY}

Within 7 days of surgery there were 28 complications in 22 grafts (10.2\%). Nine patients had a shallow anterior chamber, of which six had a leaking wound and three had aqueous leaking from a suture track. There were eight postoperative hyphaemas, seven grafts had a persistent epithelial defect, three had fibrinous uveitis, and there was one case of vitreous haemorrhage in a graft with a hyphaema.

DONOR MATERIAL

Out of 216 grafts performed, 210 donor corneas came from eye banks in the USA and Sri Lanka (mainly preserved in Optisol) and six from local donations.. The interval from donation to graft ranged from 2 to 14 days. The mean donor age was 64.6 years (range 1-92 years).

FOLLOW UP

The mean length of follow up was 27.3 months (range 1-94 months). There was no difference in the mean follow up for keratoconus and non-keratoconus grafts. Out of 203 eyes, 128 $(63.1 \%)$ were currently being seen at the eye clinic of which 111 were clear and 17 opaque. Nineteen had been discharged with an opaque graft. Patients were counted as lost to follow up if they either had a clear graft at their last visit

Table 1 Preoperative diagnoses on 216 eyes undergoing penetrating keratoplasty at Kikuyu, Kenya, 1987-92

\begin{tabular}{lrl}
\hline Diagnosis & Number & Aetiology \\
\hline Keratoconus & $108(50.0 \%)$ & \\
Corneal scar & $24(11.0 \%)$ & \\
Herpes simplex keratitis & $17(7.9 \%)$ & \\
Corneal dystrophy & $16(7.4 \%)$ & Lattice dystrophy 6 \\
& & $\begin{array}{l}\text { Macular dystrophy 3 } \\
\text { Other dystrophies 7 }\end{array}$ \\
& & Primary graft failure 5 \\
Opaque corneal graft & $14(6.5 \%)$ & $\begin{array}{l}\text { Delayed graft failure 5 } \\
\end{array}$ \\
& & Rejection 4 \\
Bullous keratopathy & $14(6.5 \%)$ & Aphakic 10 \\
& & Pseudophakic 4 \\
Trachoma & $7(3.2 \%)$ & \\
Measles & $4(1.9 \%)$ & \\
Bacterial keratitis & $4(1.9 \%)$ & \\
Other diagnoses & $8(3.7 \%)$ & \\
Total & $216(100 \%)$ & \\
\hline
\end{tabular}

Table 2 Causes of corneal graft opacification in 216 penetrating keratoplasties, Kikuyu, Kenya

\begin{tabular}{lccc}
\hline Cause of opacity & $\begin{array}{l}\text { Keratoconus } \\
(n=108)\end{array}$ & $\begin{array}{l}\text { Non-keratoconus } \\
(n=108)\end{array}$ & $\begin{array}{l}\text { Total } \\
(n=216)\end{array}$ \\
\hline Bacterial infection: & 3 & 10 & $13(6.0 \%)$ \\
$\quad$ Bacterial ulcer & 1 & 8 & 9 \\
$\quad$ Bacterial ulcer and endophthalmitis & 2 & 2 & 4 \\
Graft failure: & 7 & 6 & $13(6.0 \%)$ \\
Primary graft failure & 5 & 1 & 6 \\
Delayed graft failure & 2 & 5 & 7 \\
Graft rejection & 1 & 10 & $11(5.1 \%)$ \\
Recurrent disease: & 0 & 7 & $7(3.2 \%)$ \\
Herpes simplex keratitis & & 3 & 3 \\
Mooren's ulcer & & 3 & 1 \\
Pseudophakic bullous keratopathy & 1 & 1 & $2(0.9 \%)$ \\
Persistent epithelial defect & 0 & 1 & $1(0.5 \%)$ \\
Vitreous touch & 12 & 1 & $47(21.8 \%)$ \\
Total & & 35 & \\
\hline
\end{tabular}

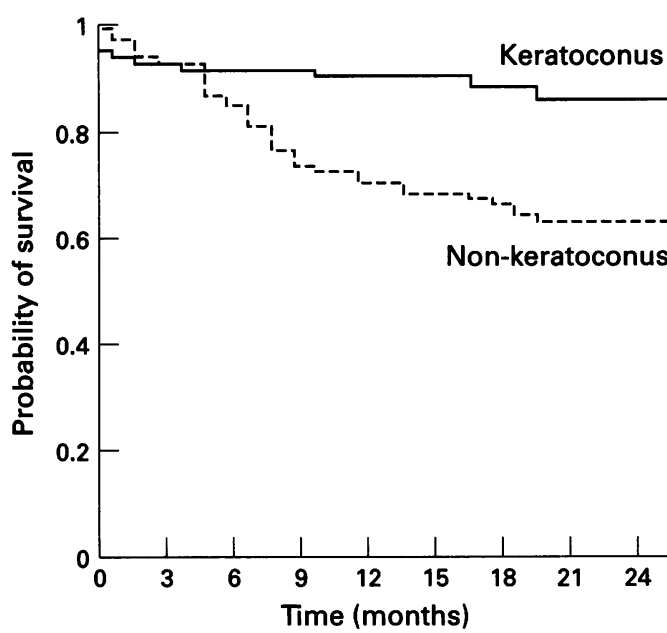

Figure 1 Probability of graft survival in 108 penetrating keratoplasties for keratoconus and 108 penetrating keratoplasties for other corneal pathologies.

and had not attended the clinic for 6 months; or in patients whose grafts had been performed over 3 years previously, if they had not attended for 12 months. Altogether 56 (27.6\%) eyes were lost to follow up, of which 35 attended for at least 6 months with a clear graft, but had not been seen during the last 6-12 months. All grafts were included in the calculation of graft survival.

\section{GRAFT SURVIVAL}

The probability of graft survival was determined using the method of Kaplan and Meier. Grafts lost to follow up were treated as censored data, and survival time was taken from their last clinic attendance. The probability of survival at 24 months among the keratoconus grafts was $87.4 \%$ (95\% CI 80.6-94.3\%) and in the non-keratoconus grafts $64.7 \%(95 \%$ CI $54.8-74.6 \%$ ). The survival curve is shown in Figure 1.

CAUSE OF GRAFT OPACIFICATION

Altogether 47 of 216 grafts $(21.8 \%)$ were known to fail in 36 of 203 eyes (17.7\%). The causes of graft opacification are given in Table 2 . Infection refers to a bacterial keratitis. This occurred in 34 grafts $(15.7 \%)$ and led to permanent graft opacity in $13(6.0 \%)$. Herpes virus infections were classified as recurrent disease. Primary graft failure was defined as graft oedema present from the time of surgery. Delayed graft failure referred to graft oedema occurring in a previously clear graft in the absence of signs of rejection. Graft rejection was defined as graft oedema, occurring in a previously clear graft, accompanied by keratic precipitates and/or uveitis.

\section{REJECTION}

There were 54 episodes of rejection in 46 grafts of 43 patients (Table 3 ). Eleven grafts $(5.1 \%)$ became permanently opaque as a result of graft rejection. A total of 21 rejection episodes occurred in keratoconus grafts, one of which led to permanent graft failure, and 33 rejection episodes occurred in non-keratoconus grafts, of which 10 led to permanent graft failure. An episode of graft rejection was more likely to 
Table 3 Graft rejection in 216 penetrating keratoplasties, Kikuyu, Kenya

\begin{tabular}{lccc}
\hline Diagnosis & Total & $\begin{array}{l}\text { Number with graft } \\
\text { rejection }\end{array}$ & $\begin{array}{l}\text { Number with graft } \\
\text { failure due to rejection }\end{array}$ \\
\hline Trachoma & 7 & $4(57.1 \%)$ & $1(14.3 \%)$ \\
Bacterial keratitis & 4 & $2(50.0 \%)$ & $0(0 \%)$ \\
Graft failure & 14 & $6(42.9 \%)$ & $2(14.3 \%)$ \\
Corneal scar & 24 & $7(29.2 \%)$ & $3(12.5 \%)$ \\
Measles & 4 & $1(25.0 \%)$ & $1(25 \%)$ \\
Corneal dystrophy & 16 & $3(18.8 \%)$ & $0(0 \%)$ \\
Keratoconus & 108 & $19(17.6 \%)$ & $1(0.9 \%)$ \\
Other & 8 & $1(12.5 \%)$ & $1(12.5 \%)$ \\
Herpes simplex keratitis & 17 & $2(11.8 \%)$ & $1(5.9 \%)$ \\
Bullous keratopathy & 14 & $1(7.7 \%)$ & $1(7.1 \%)$ \\
Total & 216 & $46(21.3 \%)$ & $11(5.1 \%)$ \\
\hline
\end{tabular}

lead to permanent graft failure in nonkeratoconus grafts than in keratoconus grafts (Fisher's exact test, $\mathrm{p}=0.02$ ). The mean time to first rejection in grafts for keratoconus was 9.9 months, and 12.0 months in grafts for other corneal pathologies.

FURTHER OPERATIONS

A total of 47 grafts required further surgery (not including repeat penetrating keratoplasty), of which $22(46.8 \%)$ became opaque. Eleven patients required resuturing of the graft following traumatic wound dehiscence of which 10 were in keratoconus eyes. Six patients required trabeculectomy and four had cataract extractions.

\section{VISUAL ACUITY}

The preoperative visual acuities in both keratoconus and non-keratoconus eyes are shown in Table 4. Non-keratoconus eyes were more likely to have a preoperative visual acuity of $<3 / 60$ than keratoconus eyes $(75.7 \%$ versus $\left.55.0 \%, \chi^{2}=9.6, p<0.01\right)$. Out of the total of 203 eyes undergoing keratoplasty, $176(86.7 \%)$ had a preoperative visual acuity of less than 6/60.

Postoperatively 109 eyes (51.7\%) had a latest visual acuity of $6 / 18$ or better (Table 4 ) of which 73 were in keratoconus eyes, and 36 were in non-keratoconus eyes $(73.0 \%$ versus $\left.35.0 \%, \chi^{2}=29.5, \mathrm{p}<0.001\right)$. Fifty seven eyes $(28.1 \%)$ had a postoperative visual acuity of less than $6 / 60$, of which 50 were in non-

Table 4 Pre-and postoperative visual acuities in 100 keratoconus and 103 non-keratoconus eyes undergoing penetrating keratoplasty at Kikuyu Hospital

\begin{tabular}{lccccc}
\hline & Preoperative & & & \multicolumn{2}{l}{ Postoperative } \\
\cline { 2 - 3 } \cline { 5 - 6 } Acuity & Keratoconus & Non-keratoconus & & Keratoconus & Non-keratoconus \\
\hline $6 / 6-6 / 18$ & $0(0 \%)$ & $0(0 \%)$ & & $73(73 \%)$ & $36(35.0 \%)$ \\
$<6 / 18-6 / 60$ & $18(18 \%)$ & $9(8.7 \%)$ & & $20(20 \%)$ & $17(16.5 \%)$ \\
$<6 / 60-3 / 60$ & $27(27 \%)$ & $16(15.5 \%)$ & & $2(2 \%)$ & $7(6.8 \%)$ \\
$<3 / 60-$ NPL & $55(55 \%)$ & $78(75.7 \%)$ & & $5(5 \%)$ & $43(41.7 \%)$ \\
Total & $100(100 \%)$ & $103(100 \%)$ & & $100(100 \%)$ & $103(100 \%)$ \\
\hline
\end{tabular}

Table 5 Pre-and postoperative visual status (acuity in better eye) of 89 keratoconus and 97 non-keratoconus patients undergoing penetrating keratoplasty at Kikuyu Hospital

\begin{tabular}{llllll}
\hline & Preoperative & & & \multicolumn{2}{c}{ Postoperative } \\
\cline { 2 - 3 } \cline { 5 - 6 } Acuity & Keratoconus & Non-keratoconus & & Keratoconus & Non-keratoconus \\
\hline $6 / 6-6 / 18$ & $22(24.7 \%)$ & $10(10.3 \%)$ & & $69(77.5 \%)$ & $37(38.1 \%)$ \\
$<6 / 18-6 / 60$ & $38(42.7 \%)$ & $27(27.8 \%)$ & & $16(18.0 \%)$ & $24(24.7 \%)$ \\
$<6 / 60-3 / 60$ & $18(20.2 \%)$ & $13(13.4 \%)$ & & $3(3.4 \%)$ & $11(11.3 \%)$ \\
$<3 / 60-$ NPL & $11(12.4 \%)$ & $47(48.5 \%)$ & & $1(1.1 \%)$ & $25(25.7 \%)$ \\
Total & $89(100 \%)$ & $97(100 \%)$ & & $89(100 \%)$ & $97(100 \%)$ \\
\hline
\end{tabular}

Table 6 Change in visual status * 186 patients undergoing keratoplasty, Kikuyu, Kenya

\begin{tabular}{llll}
\hline & Better & Same & Worse \\
\hline Keratoconus & $55(61.8 \%)$ & $34(38.2 \%)$ & 0 \\
Non-keratoconus & $41(42.3 \%)$ & $53(54.6 \%)$ & $3(3.1 \%)$ \\
Total & $96(51.6 \%)$ & $87(46.8 \%)$ & $3(1.6 \%)$ \\
\hline$\star$ Visual &
\end{tabular}

$\star$ Visual status = visual acuity in the better eye.

keratoconus eyes and seven in keratoconus eyes $\left(48.5 \%\right.$ versus $\left.7 \%, \chi^{2}=37.9, p<0.001\right)$. The non-keratoconus eyes with severe scarring and visual acuity less than $3 / 60$ tended to do badly.

\section{VISUAL RECOVERY}

A total of 118 eyes (79 keratoconus, 39 non-keratoconus) achieved a best visual acuity postoperatively of $6 / 18$ or better (of which nine subsequently deteriorated). The average time to $6 / 18$ was 7.2 months for keratoconus eyes, and 6.7 months for non-keratoconus eyes.

VISUAL STATUS

The preoperative and latest postoperative visual status of both keratoconus and nonkeratoconus patients is shown in Table 5 . Before surgery, 58 patients were blind $(<3 / 60$ in the better eye). Of these the majority $(81 \%)$ were non-keratoconus patients.

Postoperatively, $26(14.0 \%)$ patients were blind (non-keratoconus $25.7 \%$ versus keratoconus $1.1 \%$ ). A total of 34 patients who were blind preoperatively improved so that they were no longer blind after surgery. However, two patients who were not blind before surgery had become blind at their last follow up visit. Therefore, from a total of 216 penetrating keratoplasties performed, there was a net reduction in the number of blind patients of 32.

The number of patients with normal vision increased by 74, from 32 to 106 . Most of these (77.5\%) were keratoconus patients. Keratoconus patients were more likely than nonkeratoconus patients to achieve normal visual status postoperatively $(77.5 \%$ versus $38.1 \%$, $\chi^{2}=29.3, \mathrm{p}<0.001$ ).

Changes in visual status are shown in Table 6 . Ninety six patients $(51.6 \%)$ had an improvement in their visual status and three patients (1.6\%) had a worse visual status postoperatively. Of the keratoconus patients, $61.8 \%$ showed some improvement in their visual status as opposed to $42.3 \%$ of the nonkeratoconus patients $\left(\chi^{2}=7.1, \mathrm{p}<0.01\right)$

ASTIGMATISM

Postoperative astigmatism was worse in keratoconus grafts. The mean cylinder was $4.2 \mathrm{D}$ in the keratoconus eyes and $2.7 \mathrm{D}$ in nonkeratoconus eyes.

POOR VISUAL OUTCOME

Fifty seven operated eyes had a latest visual acuity of $<6 / 60$ (seven keratoconus and 50 non-keratoconus). The causes of the poor visual acuities are shown in Table 7 . Graft opacification accounted for $54.4 \%$ and glaucoma $14.0 \%$. Retinal disease was the cause in 
Table 7 Causes of postoperative visual acuity less than 6/60, in 203 eyes undergoing keratoplasty, Kikuyu, Kenya

\begin{tabular}{lll}
\hline Cause of low vision & $\begin{array}{l}\text { Non-keratoconus } \\
(n=103)\end{array}$ & $\begin{array}{l}\text { Keratoconus } \\
(n=100)\end{array}$ \\
\hline Graft opacity & 27 & 4 \\
Glaucoma & 8 & 0 \\
Retinal disease & 5 & 1 \\
Amblyopia & 3 & 1 \\
Cataract & 3 & 0 \\
Not known & 4 & 1 \\
Total & $50(48.5 \%)$ & $7(7 \%)$ \\
\hline
\end{tabular}

$10.5 \%$ and included two eyes with cystoid macular oedema, two with hereditary maculopathy, and one each with albinism and optic atrophy.

\section{Discussion}

AGE

The average age of grafting patients with keratoconus was $\mathbf{1 7 . 8}$ years which is younger than reported in other series. ${ }^{8}$ This may be due to the shortage of facilities in Kenya for fitting hard contact lenses. It is also possible that keratoconus may have an earlier onset in this population, owing to the high prevalence of vernal conjunctivitis.

\section{DIAGNOSIS}

The most frequent diagnosis was keratoconus, which was the indication for grafting in $50 \%$ of the patients. The proportion of grafts performed for herpes simplex keratitis $(7.8 \%)$ is higher than in recent series from Western countries, ${ }^{9}$ reflecting the greater severity of the disease in Africa, ${ }^{10}$ and the relative unavailability of effective treatment. It is striking that only 11 out of 216 grafts were performed for the major causes of corneal blindness in Africameasles and trachoma. The grafts that were performed for measles or trachoma did badly, as six out of the 11 grafts became opaque. This suggests that penetrating keratoplasty has a very limited role in eliminating blindness from measles, vitamin A deficiency, and trachoma in Africa.

DONOR MATERIAL

It was encouraging to see that out of 210 grafts using overseas material, there were only six cases $(3 \%)$ of primary graft failure. This confirms that it is possible to send donor corneas thousands of kilometres from their place of origin.

GRAFT SURVIVAL

Overall survival rates were lower than those reported from developed countries. ${ }^{11-14}$ In most of these series, the 2 year survival rate for keratoconus grafts was well over $90 \%$ compared with $87 \%$ in our series. Among the keratoconus grafts, the younger age of the patients, and the coexisting severe vernal disease may play a part in reducing graft survival. If the five keratoconus grafts that failed because of primary graft failure are excluded, the 2 year survival improves to $91.7 \%$. The overall rate of $64.7 \%$ survival of non-keratoconus grafts at 2 years is lower than would be expected in an industrialised country.
The major reason for the poorer prognosis is inadequate follow up. Nearly one third of patients with corneal grafts that were clear at their last follow up visit are no longer attending the clinic. Failure to attend for follow up, despite intensive preoperative counselling, is due to the cost involved in travelling to attend the clinic, and different cultural attitudes to health and disease leading to poor patient compliance.

\section{GRAFT FAILURE}

Suppurative keratitis was the leading cause of graft failure. The incidence of infection $(15.7 \%)$ is much higher than reported in the UK. ${ }^{15}$ Suppurative keratitis was associated with loose sutures, increasing age, and preoperative diagnosis of bacterial keratitis or trachoma. Secondary endothelial failure often followed traumatic wound dehiscence or wound leaks. Acute rejection was more likely to lead to graft failure in non-keratoconus grafts than in keratoconus grafts. All patients received topical steroids for at least 6 months after surgery.

VISUAL ACUITY

The preoperative visual acuities tended to be worse in the non-keratoconus eyes. This may reflect the fact that keratoconus rarely reduces the vision to hand motions. It is also likely that there is some bias, as the known better prognosis for keratoconus grafts probably encouraged earlier surgery in these patients. The final visual acuities are lower than reported for most other series, particularly for keratoconus eyes. ${ }^{11} 12$ This is partly because of the higher graft failure rate in our series, but may also be due to the fact that in other series $20-37 \%$ of the patients were at least partly reliant on contact lens correction in order to obtain good visual acuity. ${ }^{11}$ In a large multicentre prospective study of corneal grafts in the UK, $47 \%$ of eyes at 3 months and $61 \%$ at 12 months had a visual acuity of $6 / 18$ or better ${ }^{12}$; this compares with $73 \%$ for keratoconus eyes and $35 \%$ for non-keratoconus eyes in the present study. The latest visual acuity in the operated eye was worse than the vision in the unoperated eye in $13.8 \%$ of eyes. This compares with $48.3 \%$ in the series from Australia. ${ }^{16}$ This figure is likely to be an important factor in determining patient satisfaction with his or her graft.

ASTIGMATISM

We found that the mean astigmatism was significantly greater in keratoconus eyes than non-keratoconus eyes with an average astigmatism of 4.2 $\mathrm{D}$ for the keratoconus patients. Less than a quarter $(23.3 \%)$ of all eyes had a cylinder greater than 5 dioptres. As contact lenses are expensive and difficult to obtain in Kenya, astigmatism was usually managed with spectacles.

POOR VISUAL OUTCOME

Fifty seven eyes (28\%) had a postoperative visual acuity of less than $6 / 60$. The commonest cause of poor vision was graft opacity ( 31 of 57 eyes). This indicates that the most effective way of improving visual outcome will be to improve 
graft survival. The causes of poor acuity with a clear graft are similar to those reported in other series $^{8}$ although the numbers affected are proportionately greater.

VISUAL STATUS

The non-keratoconus patients were much more likely to be bilaterally blind preoperatively than the keratoconus patients. Only one keratoconus patient remained blind after penetrating keratoplasty, but 25 (25.7\%) of the non-keratoconus patients were still blind, with a vision of $<3 / 60$ in their better eye. After the 216 penetrating keratoplasties in this series, the number of blind patients was reduced by 32 - that is, an average of one in seven corneal grafts restored sight to a blind person. It is currently estimated that six million people are blind from corneal disease. ${ }^{4}$

Compared with cataract surgery, penetrating keratoplasty is relatively ineffective at restoring sight to blind patients; however, it does result in an improved visual status in the majority of patients, which can have far reaching socioeconomic effects. Consider a teenager with keratoconus and vision of $6 / 60$ and $2 / 60$. $\mathrm{He}$ will be having serious difficulties continuing his education in a normal African school. If his vision improves to $6 / 12$ in one eye following keratoplasty, he will be able to stay in a normal school and education system, with improved prospects for himself, and considerable savings for his community.

\section{Conclusions}

Penetrating keratoplasty in Africa is possible and, with care and attention, clear grafts can be obtained in many patients. We suggest that penetrating keratoplasty has a definite role in the treatment of keratoconus, which is nonpreventable, visually disabling, usually bilateral, occurs in young people, and in which nearly three quarters of grafted eyes obtained a postoperative visual acuity of $6 / 18$ or better. In non-keratoconus corneal disease, we recommend that surgery should only be considered if the fellow eye has a vision of less than $6 / 60$ and the eye for surgery is suitable for penetrating keratoplasty.

We advocate the development of selected tertiary centres for penetrating keratoplasty in
Africa. These centres should focus their efforts on grafting for keratoconus and other corneal dystrophies, and should seek to change cultural attitudes to tissue donation. In view of the limited role and poor results of penetrating keratoplasty in corneal scarring due to trachoma, vitamin A deficiency, and measlescurrently the major causes of corneal blindness in Africa-priority must still be given to community health programmes including measles immunisation, improved nutrition, trachoma control, and eyelid surgery for trichiasis.

We wish to acknowledge the assistance of Dr S Watson in data collection, and the financial support of Christoffel Blindenmission, Germany.

1 Holland S. How do we restore and maintain a clear cornea in a poor rural village? Refract Corneal Surg 1991;7:417-8. 2 Foster A. Patterns of blindness. Duane's clinical ophthalmology. Philadelphia: Lippincott, 1991;5: Chap 53.

3 Whitfield R, Schwab L, Ross-Degnan D, Steinkuller PG, Smartwood J. Blindness and Disease in Kenya: ocular status survey results from the Kenya Rural Blindness Project. Br f Ophthalmol 1989;73:82-7.

4 Foster A, Gilbert C. Community efforts in the reduction of corneal blindness in developing countries. Refract Corneal Surg 1991;7:445-8.

5 Foster A, Yorston D. Corneal ulceration in Tanzanian children: relationship between measles and vitamin A deficiency. Trans Roy Soc Trop Med Hyg 1992;86:454-5.

6 Taylor HR, West SK, Mmbaga BB, Katala SJ, Turner V, Lynch $\mathrm{M}$, et al. Hygiene factors and increased risk of trachoma in central Tanzania. Arch Ophthalmol 1989;107: 1821-5.

7 Strategies for the Prevention of Blindness in National Programmes. Geneva: World Health Organisation, 1984.

8 Kirkness CM, Ficker L, Steele ADMcG, Rice NSC. The success of penetrating keratoplasty for keratoconus. Eye 1990;4:673-88.

9 Sharif W, Casey TA. Changing indications for penetrating keratoplasy, 1971-1990. Eye 1993;7:485-8

10 Yorston D, Foster A. Herpetic keratitis in Tanzania: association with malaria. Br $\mathcal{f}$ Ophthalmol 199;76:582-5.

11 Paglen PG, Fine M, Abbott RL, Webster RG. The prognosis for keratoplasty in keratoconus, Ophthalmology 1982;89: $651-4$

12 Vail A, Gore SM, Bradley BA, Easty DL, Rogers CA. Corneal graft survival and visual outcome: a multi-centre study. Ophthalmology 1994;101:120-7.

13 The Collaborative Corneal Transplantation Studies (CCTS). Effectiveness of histocompatibility matching in high risk corneal transplantation. Arch Ophthalmol 1992; 110:1392-403.

14 Kirkness CM, Ezra E, Rice NSC, Steele ADMcG. The success and survival of repeat corneal grafts. Eye 1990;4: $58-64$.

15 Bates AK, Kirkness CM, Ficker LA, Steele ADMcG, Rice NSC. Microbial keratitis after penetrating keratoplasty. Eye 1990;4:74-8

16 Williams KA, Ash JK, Pararajasegaram P, Harris S, Coster DJ. Long-term outcome after corneal transplantation. Ophthalmology 1991;98:651-7. 